hep-th/0205007

April 2002

\title{
Notes on Periodic Solitons
}

\author{
Ioannis Bakas and Christos Sourdis \\ Department of Physics, University of Patras, \\ GR-26500 Patras, Greece \\ bakas@ajax.physics.upatras.gr, \\ sourdis@pythagoras.physics.upatras.gr
}

\begin{abstract}
We consider static solutions of the sine-Gordon theory defined on a cylinder, which can be either periodic or quasi-periodic in space. They are described by the different modes of a simple pendulum moving in an inverted effective potential and correspond to its libration or rotation. We review the decomposition of the solutions into an oscillatory sum of alternating kinks and anti-kinks or into a monotonic train of kinks, respectively, using properties of elliptic functions. The two sectors are naturally related to each other by a modular transformation, whereas the underlying spectral curve of the model can be used to express the energy of the static configurations in terms of contour integrals à la Seiberg-Witten in either case. The stability properties are also examined by means of supersymmetric quantum mechanics, where we find that the unstable configurations are associated to singular superpotentials, thus allowing for negative modes in the spectrum of small fluctuations.
\end{abstract}

\footnotetext{
${ }^{*}$ Contribution to the proceedings of the RTN European network conference "Quantum Structure of Space-time and the Geometric Nature of Fundamental Interactions" held in Corfu in September 2001; to be published in a special volume of Fortschritte der Physik edited by C. Kounnas, D. Lüst and S. Theisen.
} 


\section{Introduction}

Solitons arise as static configurations of two-dimensional scalar field theories with potentials having more than one degenerate minima. In a typical situation, where the potential $U(\phi)$ is derived from a superpotential $W(\phi)$ that is conveniently normalized as $U(\phi)=1 / 2\left(\partial_{\phi} W(\phi)\right)^{2}$, the energy functional of static configurations is bounded from below à la Bogomol'nyi

$$
E_{\text {stat }}=\int_{-\infty}^{+\infty} d x\left(\frac{1}{2}\left(\phi^{\prime}(x)\right)^{2}+U(\phi)\right) \geq \pm(W(+\infty)-W(-\infty))
$$

as the space extends over $R$. The lower bound provides the energy of static configurations that satisfy the first order equations

$$
\phi^{\prime}(x)= \pm \frac{\partial W}{\partial \phi},
$$

which also solve the second order equations of motion by construction. These are the defining equations for the solitons of the model provided that they extended between different degenerate vacua as $x$ ranges over space from $-\infty$ to $+\infty$, so that $W(+\infty) \neq$ $W(-\infty)$. Kinks correspond to solutions of the Bogomol'nyi equation with plus sign, whereas anti-kinks to minus sign. The notion also extends easily to multi-component scalar field models in two space-time dimensions with degenerate vacua.

The construction of periodic solitons poses an interesting problem when the theory is defined on a two-dimensional cylinder $R \times S^{1}$, as the spatial dimension is compact and the Bogomol'nyi bound is identically zero. Therefore, if one is interested in finding periodic solutions of the first order equation $\phi^{\prime}(x)= \pm \partial_{\phi} W(\phi)$ (or its generalizations for multi-component models), there will be no choice of the integration constants that amounts to non-trivial solutions of the same equations having a periodic structure, $\phi(x+$ $L)=\phi(x)$, other than the constant configurations sitting at the bottom of the potential with zero energy. Recall also that the physical meaning of the integration constants of Bogomol'nyi equations is provided by the location of the solitons in $x$, as it is done in non-compact space once and for all, and leaves no room for the size of the space itself, i.e., its period L, to fit into the available moduli. Following this simple observation, it turns out that the notion of periodic solitons is ill-defined for the class of two-dimensional scalar models under consideration, as no such solutions seem to be possible. We also note for completeness that in the presence of discrete symmetries in the model, for instance $Z_{2^{-}}$ invariance under $\phi \rightarrow-\phi$, one might also consider configurations with twisted boundary conditions over $S^{1}, \phi(x+L)=-\phi(x)$. Furthermore, for models with non-trivial topology in field space, it is also possible to have configurations with non-trivial winding number over $S^{1}, \phi(x+L)=\phi(x)+2 \pi$ and so on. It is straightforward to see that the no-go theorem stated above also extends to such topological sectors and hence the existence of periodic solutions of Bogomol'nyi equations remains impossible in its generality.

There are two possibilities one might subsequently entertain in order to make the notion of "periodic solitons" more precise and meaningful. The first one is to shift the 
vacuum energy density of the theory by an appropriately chosen constant so that the modified potential $\tilde{U}(\phi)$ can be derived from a new superpotential $\tilde{W}(\phi)$, which is not single-valued but its derivatives are single-valued functions. This results into a modified form of Bogomol'nyi equations, namely $\phi^{\prime}(x)= \pm \partial_{\phi} \tilde{W}(\phi)$, which are still first order and can support non-trivial periodic solutions that also satisfy the same second order classical equations of motion of the theory by construction. According to this possibility, the completion of the perfect square term in the energy functional of static configurations is done differently for theories defined over compact space in order for the boundary term to compute their energy correctly. Note, however, there is no guarantee that the periodic solitons defined in this fashion are always stable under small fluctuations, as for the case of ordinary solitons on $R$. The second possibility is to rely on the integrability properties of many two-dimensional field theory models that admit solitons on the real line and device a suitable superposition principle for constructing exact solutions by summing up trains of ordinary kinks or anti-kinks whose centers are equally spaced on the real line using a uniform length scale $L$. Despite the non-linearity of the models, such superposition principle is always possible in integrable systems and leads to periodic solutions of the second order classical equations of motion that can also be named periodic solitons by their construction.

Although these two possibilities appear to be different in nature, they turn out to be equivalent in several field theory models of current interest like sine-Gordon, $\lambda \phi^{4}$, etc that possess degenerate vacua, thus leading to an unambigious definition of the notion of periodic solitons in such two-dimensional models. Here, we will only review some aspects of the construction and the basic properties of periodic solitons arising in the sine-Gordon model and leave further details and generalizations to future publications [1]. We will also spell out some connections with the Seiberg-Witten theory of BPS states in four-dimensional supersymmetric gauge theories as application of our results.

\section{Sine-Gordon model on $R \times S^{1}$}

Consider the sine-Gordon theory with Lagrangian density

$$
\mathcal{L}=\frac{1}{2}\left(\partial_{\mu} \phi\right)\left(\partial^{\mu} \phi\right)-U(\phi) ; \quad U(\phi)=1+\cos \phi
$$

defined on a two-dimensional cylindrical space-time $R \times S^{1}$. We are interested in the construction of static configurations satisfying the classical equations of motion

$$
\phi^{\prime \prime}(x)=\frac{\partial U}{\partial \phi}
$$

with strictly periodic boundary conditions on the spatial direction

$$
\phi(x+L)=\phi(x)
$$


with period $L$; later we will also take advantage of the non-trivial topology in the field space of the model and consider solutions in the winding sector, i.e., quasi-periodic solutions with $\phi(x+L)=\phi(x)+2 \pi$. We proceed by multiplying both sides of the equation of motion with $\phi^{\prime}(x)$, which upon integration yields

$$
\frac{1}{2}\left(\phi^{\prime}(x)\right)^{2}-U(\phi)=-C
$$

where $C$ denotes the corresponding integration constant. We can think of this equation as describing a constant "energy" condition for a point particle that moves in an inverted potential $-U(\phi)$ with respect to an effective "time" $x$ [2]. This is an appropriate point of view for constraining the values of $C$ associated with strictly periodic solutions as they correspond to bounded effective motion, thus leading to $-2 \leq-C \leq 0$; later we will also let $-C \geq 0$ to account for quasi-periodic solutions in the winding sector of the model that correspond to unbounded effective motion. In the language of a classical pendulum, which is natural to consider for the sine-Gordon potential, these two different cases correspond to the modes of libration and rotation, respectively.

It is convenient to parametrize for the present purposes the parameter $C$ as

$$
C=2\left(1-k^{2}\right) ; \quad 0 \leq k \leq 1
$$

whereas $k^{\prime}$ will denote later the complementary modulus, $k^{2}+k^{\prime 2}=1$. Then, one easily finds the static solution

$$
\phi(x)= \pm 2 \sin ^{-1}\left(k \operatorname{sn}\left(x+x_{0} ; k\right)\right)
$$

with $L$ being identified with the real period of the Jacobi elliptic function $\operatorname{sn}(x+L ; k)=$ $\operatorname{sn}(x, k)$, i.e.,

$$
L=4 m K(k) ; \quad m \text { positive integer, }
$$

where $k$ serves as the modulus of the associated complete elliptic integral of the first kind, $K(k)$. The two possible signs arise from taking the square root before integrating the resulting first order equation and they are compatible with the discrete symmetry $\phi \rightarrow-\phi$ of the sine-Gordon model. Also, the integration constant $x_{0}$ determines the location of the configuration and can be set equal to zero without loss of generality.

It is also interesting to consider two special limits that arise for $k=1$ (the point particle being at the verge of becoming unbounded) and $k=0$ (the point particle sitting at the bottom of the effective potential $-U(\phi))$ :

$\underline{k=1}$ : in this case we have $\operatorname{sn}(x ; k=1)=\tanh x$ and therefore the static solution (with plus sign) becomes

$$
\phi(x)=2 \sin ^{-1}(\tanh x) \equiv 4 \tan ^{-1} e^{x}-\pi,
$$

which is the usual kink solution centered at $x=0$ ( $x_{0}$ more generally) that interpolates monotonically between $\phi=-\pi$ for $x \rightarrow-\infty$ and $\phi=\pi$ for $x \rightarrow+\infty$ in the sine-Gordon potential; in this case the spatial dimension $S^{1}$ decompactifies to $R$ as $K(1)$, and hence the period, becomes infinite. Note that the anti-kink solution will emerge in this limit, if we take the opposite sign in the general periodic expression. 
$\underline{k=0}$ : in this case we have $\operatorname{sn}(x ; k=0)=\sin x$ and therefore we simply obtain the sphaleron configuration

$$
\phi(x)=0
$$

which represents a constant configuaration at the top of the sine-Gordon potential $U(\phi)$.

Thus, for generic values of the modulus $0 \leq k \leq 1$, we have a periodic solution whose particular properties are worth studying, as they extrapolate from the stable structure of a monotonic kink to the highly unstable structure of a sphaleron. We will also learn some lessons from this study later, by using two different methods based on the direct computation of the fluctuation spectrum as well as on methods of supersymmetric quantum mechanics.

Next, we illustrate a superposition principle underlying the periodic solution of the non-linear sine-Gordon equation for generic values of $k$. Consider the usual kink solutions of the model written in a convenient equivalent form

$$
\phi_{ \pm}(x)=2 i \log \frac{1 \mp i e^{x}}{1 \pm i e^{x}}-\pi
$$

where \pm correspond to a kink or an anti-kink configuration on $R$. Then, using standard identities from the theory of Jacobi elliptic functions, it is fairly straightforward to decompose the static periodic solution $\phi(x)=2 \sin ^{-1}(k \operatorname{sn}(x ; k))$ as follows:

$$
\begin{aligned}
\phi(x) & =\sum_{n=-\infty}^{+\infty}\left(2 i \log \frac{1-i \exp \left(\frac{\pi}{2 K\left(k^{\prime}\right)}(x-4 n K(k))\right)}{1+i \exp \left(\frac{\pi}{2 K\left(k^{\prime}\right)}(x-4 n K(k))\right)}-\pi\right)+ \\
& +\sum_{n=-\infty}^{+\infty}\left(2 i \log \frac{1+i \exp \left(\frac{\pi}{2 K\left(k^{\prime}\right)}((x+2 K(k))-4 n K(k))\right)}{1-i \exp \left(\frac{\pi}{2 K\left(k^{\prime}\right)}((x+2 K(k))-4 n K(k))\right)}-\pi\right) .
\end{aligned}
$$

Therefore, the periodic solution is composed of an alternating sum of kinks and anti-kinks separated from each other by $2 K(k)$, which produce an oscillatory train solution with net period $4 K(k)$ (see also [3]).

\section{Spectrum of small fluctuations}

The stability properties of a classical solution can be investigated in general by studying the spectrum of small fluctuations around it. It is well known that the relevant Schrödinger problem for the spectrum is given by

$$
\left(-\frac{d^{2}}{d x^{2}}+V(x)\right) \psi_{k}(x)=\omega_{k}^{2} \psi_{k}(x),
$$

where the Schrödinger potential is

$$
V(x)=\left.\frac{\partial^{2} U}{\partial \phi^{2}}\right|_{\phi_{\mathrm{cl}}(x)}
$$


for any given classical field configuration $\phi_{\mathrm{cl}}(x)$. Thus, stability is ensured by the absence of tachyonic (negative) modes. The spectrum can be computed directly, when this is possible, but in many typical situations the Schrödinger potential assumes a form as in supersymmetric quantum mechanics, i.e.,

$$
V(x)=\mathcal{W}^{2}(x)-\mathcal{W}^{\prime}(x)
$$

where $\mathcal{W}(x)$ is the corresponding superpotential [4]. In these cases one concludes automatically that the spectrum is bounded from below by zero, thus establishing the desired stability properties without any further computation. There are some exceptional circumstances, however, where such conclusions should be drawn with care in order to avoid wrong statements.

The static periodic solution of the sine-Gordon equation is expected to be unstable because of its oscillatory nature, unlike the stability of ordinary kinks which are monotonic functions. Direct computation of the spectrum in this case amounts to solving the Schrödinger problem with potential

$$
V(x)=2 k^{2} \operatorname{sn}^{2}(x ; k)-1
$$

known as the Lamé potential. It can be easily verified that it supports the following three square-integrable solutions:

$$
\begin{aligned}
& \psi(x)=\operatorname{cn}(x ; k), \quad \text { with } \omega^{2}=0, \\
& \psi(x)=\operatorname{dn}(x ; k), \quad \text { with } \omega^{2}=k^{2}-1, \\
& \psi(x)=\operatorname{sn}(x ; k), \quad \text { with } \omega^{2}=k^{2} .
\end{aligned}
$$

The second solution is clearly tachyonic for $0 \leq k<1$, which proves the instability of the given solution in agreement with our intuition (see also [2]).

On the other hand, the Lamé potential can be derived from a superpotential, as in supersymmetric quantum mechanics, with

$$
\mathcal{W}_{k}(x)=\frac{\operatorname{dn}(x ; k) \operatorname{sn}(x ; k)}{\operatorname{cn}(x ; k)} .
$$

Therefore, one would expect to have no negative modes at all by relying on the general properties of supersymmetric quantum mechanics; this, in turn, poses an interesting puzzle by itself of having instability versus supersymmetry. The aparent confict is enhanced in the limit $k=0$, where it becomes obvious, as we have

$$
V_{k=0}(x)=-1, \quad \mathcal{W}_{k=0}(x)=\tan (x)
$$

In the other limit, where $k=1$, we have

$$
V_{k=1}(x)=1-\frac{2}{\cosh ^{2} x}, \quad \mathcal{W}_{k=1}(x)=\tanh (x)
$$


and the conflict goes away; note at this end that the supersymmetric partner of $V_{k=1}(x)$ is the constant potential $V(x)=1$ with manifestly positive spectrum, as it is required for an ordinary kink on $R$.

The resolution to this paradox is provided by the simple fact that the superpotential $\mathcal{W}_{k}(x)$ is not regular everywhere in its domain of definition, for all $0 \leq k<1$. It is sufficient to note for the present problem that the elliptic function $\mathrm{cn}(x ; k)$ develops zeros for all real $x=(2 m+1) K(k)$ and hence the corresponding $\mathcal{W}_{k}(x)$ becomes singular at two points, as $x$ ranges from 0 to $4 K(k)$; for $\mathcal{W}_{k=0}(x)$, in particular, the singularity occurs at $x=\pi / 2$ and $3 \pi / 2$, as $x$ ranges from 0 to $4 K(0)=2 \pi$. It has been known for some time that the bear facts of supersymmetric quantum mechanics should be used with care under such circumstances, for the case of singular superpotentials could obstruct their validity, like the positivity of the spectrum (see [4] and references therein). Finally, we note for completeness that the static solution in the twisted sector of the model, which has anti-periodic boundary conditions $\phi(x+2 K(k))=-\phi(x)$, exhibits stability as the troublesome negative mode $\psi(x)=\operatorname{dn}(x ; k)$ has real period $2 K(k)$ and hence is moded out by the twisting [5]. Put it differently, in the language of supersymmetric quantum mechanics, the singularities of the superpotential occuring at $x=K(k)$ and $3 K(k)$ are removed from the interval obtained by twisting, as they constitute the fixed points of the $Z_{2}$ action.

\section{Spectral curve and its applications}

Next, we provide a natural algebro-geometric description of the energy of periodic static configurations of the sine-Gordon equation, which is reminiscent of the Seiberg-Witten expression for the mass of the BPS states in $N=2$ supersymmetric Yang-Mills theories for the group $S U(2)$ [6]. Along these lines we will also examine the behaviour of static solutions under the action of modular transformations of an underlying Riemann surface and show that the two different modes of evolution, namely the libration and rotation of a classical pendulum, are intimately connected to each other by them. Actually, this is not a surprise, as the underlying integrable system of Seiberg-Witten theory is the sineGordon model [7], whereas the supersymmetric BPS states of four-dimensional gauge theory are monopoles and dyons.

It is sufficient for this purpose to consider the zero curvature formulation of the static sine-Gordon equation in terms of the Nahm equations

$$
\frac{d T_{i}(s)}{d s}=\frac{1}{2} \sum_{j, k=1}^{3} \epsilon_{i j k}\left[T_{j}(s), T_{k}(s)\right] ; \quad i=1,2,3
$$

for the special choice of matrices

$$
T_{1}=-2 i\left(\cos \frac{1}{2} \phi\right) \sigma_{1}, \quad T_{2}=2\left(\sin \frac{1}{2} \phi\right) \sigma_{2}, \quad T_{3}=\frac{1}{2}\left(\frac{d \phi}{d s}\right) \sigma_{3},
$$


where $\sigma_{i}$ are the three Pauli matrices. The system of Nahm equations admits the zero curvature formulation

$$
\left[L(s), \frac{d}{d s}+M(s)\right]=0
$$

where

$$
\begin{aligned}
L & =T_{1}+i T_{2}-2 i \zeta T_{3}+\zeta^{2}\left(T_{1}-i T_{2}\right), \\
M & =-i T_{3}+\zeta\left(T_{1}-i T_{2}\right)
\end{aligned}
$$

using a spectral parameter $\zeta$. Note that the choice of Nahm matrices above yields the sine-Gordon equation for the variable $x=2 i s$,

$$
\phi^{\prime \prime}(x)+\sin \phi(x)=0
$$

as required.

Let $\eta$ denote the eigenvalue of the Lax matrix $L(s)$, which is defined by the equation

$$
\operatorname{det}(L(s)-\eta 1)=0
$$

that yields the quartic algebraic equation in the complex variable $\zeta$,

$$
\eta^{2}+1+2 \zeta^{2}\left(-\frac{1}{2}\left(\phi^{\prime}(x)\right)^{2}+\cos \phi(x)\right)+\zeta^{4}=0 .
$$

Since the Lax pair formulation of the problem implies an isospectral flow for the matrix $L$, the coefficients of the resulting polynomial in $\zeta$ have to be independent of $x$, thus providing the conservation laws of the system. In our case, using the notation adopted earlier, we have

$$
-\frac{1}{2}\left(\phi^{\prime}(x)\right)^{2}+\cos \phi(x)=C-1 \equiv 1-2 k^{2} .
$$

This defines the specral curve of the system, which is a Riemann surface of genus 1 (see also [8]).

For bounded motion in the inverted sine-Gordon potential $-U(\phi)$, i.e., for $0 \leq k<1$, the four roots of the quartic polynomial in $\zeta$ are all complex occuring in conjugate pairs

$$
k+i k^{\prime}, \quad k-i k^{\prime}, \quad-\left(k+i k^{\prime}\right), \quad-\left(k-i k^{\prime}\right) .
$$

Then, transforming to the Weierstrass normal form of the spectral curve,

$$
y^{2}=4\left(x-e_{1}\right)\left(x-e_{2}\right)\left(x-e_{3}\right) ; \quad \sum_{i=1}^{3} e_{i}=0,
$$

we find the three roots $e_{1} \geq e_{2} \geq e_{3}$ to be given by

$$
e_{1}=\frac{1}{3}\left(2-k^{2}\right), \quad e_{2}=\frac{1}{3}\left(2 k^{2}-1\right), \quad e_{3}=-\frac{1}{3}\left(1+k^{2}\right) .
$$

The energy of the periodic static configuration can be computed directed in sineGordon theory. It is convenient to shift the vacuum energy density by the constant 
$C$, thus defining a new potential $\tilde{U}(\phi)=U(\phi)-C$, which does not alter the classical equations of motion. Then, it can be easily seen that the energy of our periodic static configuration can be cast into the form

$$
E_{\text {stat }}(k)=8 \oint_{a} \frac{d x}{y}\left(x-e_{2}\right)
$$

using a contour integral over the $a$-cycle of the corresponding spectral curve, which encircles the branch cut between the roots $e_{2}$ and $e_{3}$. If we had taken the static solution in the twisted sector of the model with $\phi(x+2 K(k))=-\phi(x)$, the corresponding energy would have been half of it. Also note in this context that when the $a$-cycle shrinks to zero size (i.e., when $e_{2} \rightarrow e_{3}$ or else $k \rightarrow 0$ ), one obtains the normalized energy of the sphaleron configuration sitting at the top of the potential; likewise, when the $b$-cycle shrinks to zero size (i.e., when $e_{1} \rightarrow e_{2}$ or else $k \rightarrow 1$ ), one obtains the energy of the usual kink and anti-kink configurations in non-compact space.

At this point it is natural to consider the case of unbounded motion in more detail, which defines solutions in the winding sector of the sine-Gordon model that correspond to the rotational mode of a classical pendulum, as opposed to the strictly periodic mode of libration. It is sufficient for this purpose to consider values of the modulus $k$ bigger than 1 , in which case $-C \geq 0$. The extension to those values is naturally defined by the following transformation of the modulus $k$ and its complementary value $k^{\prime}$,

$$
\tilde{k}=\frac{1}{k}, \quad \tilde{k}^{\prime}=i \frac{k^{\prime}}{k}
$$

which results from the transformation law of the complex modulus $\tau$,

$$
\tilde{\tau}=\frac{\tau}{\tau+1} ; \quad \text { where } \tau=i \frac{K\left(k^{\prime}\right)}{K(k)} .
$$

This corresponds to the modular transformation TST, which is composed from the elementary modular transformations $T: \tau \rightarrow \tau+1$ and $S: \tau \rightarrow-1 / \tau$. As such, it amounts to a transformation of the complete elliptic integrals of the first kind, given by,

$$
K(\tilde{k})=k\left(K(k)+i K\left(k^{\prime}\right)\right), \quad K\left(\tilde{k}^{\prime}\right)=k K\left(k^{\prime}\right)
$$

and turns the four complex roots of the quartic polynomial of the spectral parameter $\zeta$ into four real roots, respectively,

$$
\pm\left(\tilde{k} \pm i \tilde{k}^{\prime}\right)= \pm\left(\frac{1}{k} \mp \frac{k^{\prime}}{k}\right)
$$

It can be readily seen that under the modular transformation $\tau \rightarrow \tilde{\tau}$, we have

$$
\exp \left(\frac{\pi}{2 K\left(k^{\prime}\right)}(x-2 n K(k))\right) \rightarrow(-1)^{n} \exp \left(\frac{\pi}{2 K\left(k^{\prime}\right)}\left(\frac{x}{k}-2 n K(k)\right)\right)
$$

and so the oscillatory train of alternating kinks and anti-kinks, with period $4 K(k)$, turns into a monotonic superposition of kinks (or anti-kinks) only. 
In effect, the Dehn twist prescribed on the torus of the corresponding Jacobi variety, where $x$ takes real values along a closed cycle, maps strictly periodic solutions into solutions belonging in the winding sector of the sine-Gordon model with real quasi-period $2 k K(k)$,

$$
\phi(x+2 k K(k))=\phi(x)+2 \pi .
$$

In fact, using the identity $\tilde{k} \operatorname{sn}(x ; \tilde{k})=\operatorname{sn}(x / k ; k)$, we can easily cast the resulting monotonic solution of superimposed kinks in closed form and verify directly that under a $2 k K(k)$-shift we obtain the desired quasi-periodic behaviour. Then, it turns out that the energy of this configuration can be written in the form

$$
\tilde{E}_{\text {stat }}(k)=4\left(\oint_{a} \frac{d x}{y}\left(x-e_{2}\right)-\oint_{b} \frac{d x}{y}\left(x-e_{2}\right)\right),
$$

using contour integration over the $a$ - and $b$-cycles of the Riemann surface with complex modulus $\tau$ having $k>1$. Of course, as before, the result is conveniently presented after shifting the vacuum energy density of the model by the constant $C$.

\section{Conclusions}

In summary, we derived a classic and intuitive result effectively stating that the libration of a pendulum can be decomposed into an alternating train of kinks and anti-kinks, whereas its rotational motion can be decomposed into a train of kinks or anti-kinks only, depending on the two possible orientations of rotation. The monotonic nature of the solution in the latter case, also ensures the absence of any negative eigenvalues from the spectrum of small fluctuations around it; clearly, the troublesome eigenvalue $k^{2}-1$ of the corresponding Lamé potential ceases to be tachyonic for $k \geq 1$, thus leading to stability. Put it differently, the superpotential $\mathcal{W}_{k}(x)$ of the associated problem in supersymmetric quantum mechanics becomes non-singular in its domain of definition after a modular transformation and, hence, the eigenvalues turn out to be bounded from below by zero. Finally, we note that the expressions for the energy of static configurations in the twisted or in the winding sector of the sine-Gordon theory coincide with the mass formula of certain BPS states in four-dimensional Seiberg-Witten theory; further details will be presented elsewhere [1].

\section{Acknowledgements}

This work was supported in part by the European research and training networks "Superstring Theory" (HPRN-CT-2000-00122) and "The Quantum Structure of Space-time" (HPRN-CT-2000-00131). The research of C.S. is also supported by a graduate student fellowship "C. Caratheodory" under the grant number 2453 from the University of Patras, which we also gratefully acknowledge. 


\section{References}

[1] I. Bakas and C. Sourdis, in preparation.

[2] N. Manton and T. Samols, Phys. Lett. B207 (1988) 179; J.-Q. Liang, H. MüllerKirsten and D. Tchrakian, Phys. Lett. B282 (1992) 105.

[3] M. G. Forest and D. McLaughlin, J. Math. Phys. 23 (1982) 1248.

[4] F. Cooper, A. Khare and U. Sukhatme, Phys. Rep. 251 (1995) 267.

[5] S. J. Avis and C. J. Isham, Proc. R. Soc. Lond. A363 (1978) 581.

[6] N. Seiberg and E. Witten, Nucl. Phys. $\underline{B 426}$ (1994) 19; Nucl. Phys. B426 (1994) 484; Nucl. Phys. B430 (1994) 485 (E).

[7] A. Gorskii, I. Krichever, A. Marshakov, A. Mironov and A. Morozov, Phys. Lett. $\underline{B 355}$ (1995) 466; E. Martinec and N. Warner, Nucl. Phys. B459 (1996) 97.

[8] P. Sutcliffe, Phys. Lett. B381 (1996) 129. 